\title{
La vocation ostensive du langage : pour une interprétation de la notion d'autonymie
}

\author{
Fasciolo, Marco \\ Université Paris 13, LDI UMR 7187 \\ marco.fasciolo@gmail.com
}

\section{Introduction}

L'histoire de l'autonymie peut être décrite comme un conflit entre deux forces théoriques : la séparation et l'unification. Considérons les mots soulignés dans les exemples (0) :

(0) a. Chat a quatre lettres.

b. Le chat a volé la saucisse.

Pour la séparation, il s'agit de deux signes linguistiques différents : autonyme en (0a) et non-autonyme en $(0 \mathrm{~b})^{1}$. Par conséquent, dans cette perspective, l'on s'interroge sur leur rapport: s'agit-t-il d'une dénomination? S'agit-t-il d'une référence? S'agit-t-il d'une représentation? Etc. Puisque la séparation distingue deux signes et s'interroge sur leur rapport, elle offre une analyse sémiotique de l'autonymie.

Pour l'unification, en revanche, en (0), il y a un seul signe linguistique chat : le signe autonyme et le signe non-autonyme sont une seule et même chose (cf. Washington, 1992:601) ${ }^{2}$. Par conséquent, l'unification analyse l'autonymie en termes de présence d'un mot et de catégorisation du mot présent. Comme J. R. Searle l'écrit :

Le mot lui-même est présenté, et ensuite, on en parle, et le fait qu'il soit ainsi utilisé, plutôt qu'employé pour référer comme il est normal, est indiqué par les guillemets. Mais dans ce cas, on ne réfère pas à ce mot, pas plus qu'il ne se réfère à lui-même (Searle 1969, 1996:119).

Dans cette perspective, les questions pertinentes sont : à quel objet sommes-nous confrontés ? Quelle est la catégorisation pertinente de l'objet auquel nous sommes confrontés? C'est la perspective que nous adoptons.

Du côté de la séparation, l'on peut placer les défenseurs de la théorie de la dénomination : par exemple, Tarski (1944), Quine (1960), Rey-Debove (1978/1997), Authier-Revuz (1995, 2003). Du côté de l'unification, l'on peut placer les auteurs utilisant des notions telles que exhibition, ostension, présentation, démonstration, identité : par exemple, Sørensen (1961), Searle (1969), Christensen (1970), Recanati (1979), Washington (1992) et Saka (1998). Cette distribution est inévitablement schématique car le même auteur peut mélanger des aspects relevant des deux pôles ${ }^{3}$. Notre proposition se situe à l'extrémité du pôle de l'unification et est basée sur trois faits.

Le premier fait est la présence du langage: les mots sont les seuls objets toujours présents dans tout champ d'indication (Prandi 2004) identifié par une situation d'énonciation concrète.

Le deuxième fait est la catégorisation multiple d'un objet: un seul et même objet présent dans une situation d'énonciation peut être conceptualisé de différentes façons. L'objet en question peut être non linguistique ou linguistique. Un exemple d'objet non linguistique : la même personne envisagée en tant que citoyen français, mammifère, corps matériel, etc. Un exemple d'objet linguistique : le même mot envisagé en tant que cri, ordre, nom commun, etc. 
Le troisième fait est la vocation ostensive du langage: les expressions linguistiques ont une aptitude naturelle à s'attacher à tout objet présent dans une situation d'énonciation. Encore une fois, les objets en question peuvent être non linguistiques ou linguistiques. Un exemple d'objet non linguistique : la surface d'une tombe sur laquelle est sculpté l'expression à la mémoire de Jean Dupont. Un exemple d'objet linguistique : l'assertion il y a du champagne dans la cave à laquelle l'on juxtapose la subordonnée si tu as une invitée. La subordonnée si tu as une invitée est à l'assertion il y a du champagne dans la cave ce que l'incision à la mémoire de Jean Dupont est à la tombe.

Les trois faits susmentionnés s'enchaînent de la façon suivante. Si les mots sont toujours présents dans n'importe quelle situation d'énonciation (présence du langage), il n'y a aucun besoin de nous référer à eux, mais l'on peut directement les indiquer et les commenter (vocation ostensive) en les catégorisant de différentes façons (catégorisation multiple). Le phénomène appelé autonymie surgit quand la vocation ostensive du langage s'exerce sur des mots catégorisés en tant qu'items lexicaux.

Le but de notre proposition est donc double. D'une part, elle vise à offrir une interprétation de l'autonymie dont aucun trait ne relève du pôle de la séparation. D'autre part, elle propose de considérer l'autonymie comme un cas particulier de la vocation ostensive susmentionnée : à savoir, la pointe de l'iceberg d'un continuum de phénomènes qui inclut motifs du dire, motifs de l'acte linguistique, etc. (cf. Prandi 2004; Gross\&Prandi 2004 ; Prandi 2006).

Les $\S \S 1 ., 2$. et 3. seront dédiés, respectivement, à présenter les notions de présence du langage, catégorisation multiple d'un objet et vocation ostensive (du langage). Sous §4., nous considérerons cinq questions classiques concernant la notion d'autonymie.

\section{Présence du langage}

La notion de présence du langage peut être envisagée comme la conjonction des trois propositions suivantes : a) les mots sont les seuls objets qui n'ont pas besoin de substituts $(\S 1.1) ; b)$ les mots ne se réfèrent pas à eux-mêmes: ils sont eux-mêmes $(\S 1.2) ; c)$ un mot-type n'est pas un concept ou un universel, mais bien un particulier ou un individu (eidétique) (\$1.3).

\subsection{Les mots n'ont pas besoin de substituts}

Considérons les exemples suivants :

(1) a. Son roman a été déchiré.

b. Son roman est plein de suspense.

(2) a. Roman est un nom commun.

b. Roman est un compliment (pour ce qu'il a écrit).

Avec les termes de Gross (2012), en (1), les prédicats déchirer et être plein de suspense sont cohérents respectivement avec un membre des classes des <concrets> et des <intrigues>, alors qu'en (2) les prédicats être un nom et être un compliment sont cohérents respectivement avec un membre de la classe des $<$ mots $>$ et des $<$ mots d'appréciation $>$. Entre ces classes il y a une différence. Les membres du premier couple doivent être dénotés car ils sont in absentia : le fait d'énoncer (1) ne nous met pas en présence du roman dont l'on parle. Les membres du second couple, en revanche, ne doivent pas être dénotés car ils sont in praesentia : le fait d'énoncer (2) nous met en présence du mot dont l'on parle. Ce point est saisi par Searle (1969:118).

Comme Prandi (2006:243) le souligne, les mots jouissent d'un privilège, car nous pouvons parler de n'importe quel objet, mais, ce faisant, il y aura toujours un type d'objet déjà présent : les mots. D'ailleurs, les mots employés sont le résultat d'un choix. Ainsi, en parlant, nous pouvons accomplir les actes illocutoires les plus variés, mais il y aura un acte que nous accomplirons toujours et pour lequel nous pourrons toujours être jugés : choisir ces mots. Si cela est vrai, quand nous parlons ou écrivons, nous 
n'avons aucun besoin de nous référer ni à nos mots, ni à leur choix, parce qu'ils sont immédiatement à disposition (cf. Fasciolo 2008:754).

Les mots sont donc les seuls objets qui n'ont pas besoin de substituts. Cette idée peut être représentée à travers la citation suivante :

\begin{abstract}
Vous souvenez-vous du sage Balnibarbi, dans les Voyages de Gulliver? Il avait décidé que, "puisque les mots ne sont que des substituts des choses, il serait plus pratique pour tous les hommes d'emporter avec eux les choses qui seraient nécessaires pour exprimer les affaires particulières dont ils auraient à discuter » Il apparut cependant qu'il y avait un inconvénient [...] Il serait difficile de parler en choses d' "une baleine ", plus embarrassant encore parler «des baleines », et pratiquement impossible de communiquer quoi que ce soit sur « toutes les baleines » ou sur « les baleines absentes » (Jakobson 1963:42).
\end{abstract}

Les mots sont les seuls objets pour lesquels la suggestion du sage Balnibarni est viable. Signalons deux remarques cohérentes avec cette conclusion.

La première remarque est qu'une langue a des mots pour des choses qui ne sont pas des mots (par exemple tigre pour les tigres) et des mots (métalinguistiques) pour des types de mots (adjectif, pronom etc.). Cependant, une langue ne peut pas avoir un mot pour le mot tigre comme elle a le mot tigre pour les tigres. La raison est simple : dans la langue, il y a les mots exactement comme, dans la savane, il y a les tigres. Cela rejoint la position défendue par Searle (1969:118-119).

La seconde remarque est la suivante. La définition du dictionnaire d'un mot non métalinguistique ne commence pas par: mot qui signifie..., mais elle décrit tout de suite le signifié. De même, la définition d'un mot métalinguistique ne commence pas par: mot qui signifie un mot qui..., mais elle décrit tout de suite le signifié : mot qui remplit cette fonction.... Dans les deux cas, le dictionnaire définit immédiatement la signification du mot; en aucun cas, il ne répète qu'il s'agit d'un mot. Pourquoi ? Parce que ce serait redondant vu que ce mot lui-même est déjà là.

\title{
1.2 Les mots ne se réfèrent pas à eux-mêmes : ils sont eux-mêmes
}

Considérons les exemples suivants :
a. Voilà un ange.
b. Voici un mot français de quatre lettres : ange.
c. 'Ange' a quatre lettres.

Imaginons qu'on emploie l'énoncé (3a) pour indiquer une jeune fille (Hélène) qui se promène sur un boulevard. Dans cette hypothèse, le référent de l'expression un ange est Hélène et il serait absurde de se demander quel est le référent de Hélène : la fille n'a pas de référent, elle est le référent de l'expression un ange. Affirmer que Ange, en (3b) ou (3c), se réfère à lui-même (ou, plus généralement, affirmer qu'il se réfère à quelque chose) est une absurdité du même ordre que d'affirmer qu'une jeune fille se réfère à elle-même (ou, plus généralement, que n'importe quel objet se réfère à lui-même).

Un boulevard et une page sont des champs déictiques (deixis ad oculos, avec la formule de Karl Bühler). Pour rendre saillant un objet dans le premier, nous pouvons utiliser un doigt ou un regard, alors que pour rendre saillant un objet dans le second, nous utilisons des locutions comme un mot français ou des stratégies graphiques comme les guillemets et l'italique. Ces dernières ne remplissent pas la fonction de transformer un signe en un autre signe, mais de le mettre en évidence, comme des clins d'œil méta-textuels. Regardons les détails.

En (3a) et (3b), il y a deux objets (Hélène et le mot ange), respectivement extralinguistique et linguistique, qui sont les référents des expressions un ange et un mot français de quatre lettres. En (3c), en revanche, il y a un objet linguistique (le mot ange), qui n'est le référent d'aucune expression linguistique. 
En (3a), Hélène est présente sur un boulevard et indiquée par l'expression un ange; en (3b), le mot ange est présent sur le papier et indiqué par l'expression un mot français de quatre lettres; en (3c), le mot ange est présent sur le papier et mis en évidence par l'italique ou les guillemets. En (3b) et en (3c), le mot ange, en tant qu'objet présent sur le papier, est lui-même et il ne renvoie à rien d'autre : exactement comme Hélène, en tant que fille présente sur le boulevard, est elle-même et elle ne renvoie à rien d'autre.

Un corollaire est que l'autonymie n'est pas un cas d'auto-référence. Considérons un énoncé tel que (4) :

Cette phrase est écrite en italique.

Spontanément, l'énoncé (4) sera interprété d'une façon auto-référentielle : dans cette interprétation, d'ailleurs, il exprime une proposition vraie. Cependant, l'énoncé (4) n'est pas un cas d'autonymie. L'autonymie n'est donc pas un phénomène auto-référentiel : en fait, elle n'est pas du tout un phénomène référentiel.

\subsection{Un mot-type n'est pas un universel, mais bien un individu}

Comparons les exemples (5) :

$$
\begin{array}{ll}
\text { a. C'est la même baleine. } & {[\mathrm{Ce}=\text { deux baleines dans un aquarium }]} \\
\text { b. C'est le même mot. } & {[\mathrm{Ce}=\text { deux occurrences ou tokens du mot type baleine }]}
\end{array}
$$

Supposons que le pronom $c e$ en $(5 \mathrm{a})$ se réfère à deux baleines dans un aquarium et que le pronom $c e$ en (5b) se réfère à deux occurrences (tokens) du même mot (type) baleine. Dans cette hypothèse, les prédicats nominaux en (5a) et (5b) s'avèrent différents. En (5a), baleine sera interprété comme type (au sens usuel) ou espèce de baleine : le prédicat nominal en (5a) assigne deux individus à la même classe. En (5b), en revanche, mot ne sera pas interprété comme type (au sens usuel) ou espèce de mot : le prédicat nominal en (5b) établit l'identité matérielle entre deux individus. Le fait qu'en (5a) baleine est interprété comme espèce de baleine suggère qu'il y a le concept de baleine ; le fait qu'en (5b) mot ne peut pas être interprété comme espèce ou type (au sens usuel) de mot suggère qu'il n'y a pas le concept (ou l'universel) du mot baleine ${ }^{4}$. Le mot-type baleine n'est donc pas un concept ou un universel.

Considérons les questions (6) suivantes :

(6) a. Pourriez-vous me montrer un exemplaire de baleine, s'il vous plaît?

b. Pourriez-vous me montrer un exemple de nom, s'il vous plaît?

c. Pourriez-vous me montrer un échantillon de marbre, s'il vous plaît?

Les questions (6) sont heureuses, mais les questions (7) ne le sont pas :

(7) a. ??Pourriez-vous me montrer un exemple de la Vénus de Milo, s'il vous plaît?

b. ??Pourriez-vous me montrer un exemple de 'baleine', s'il vous plaît? ${ }^{5}$

Offrir un exemple d'un mot est absurde tout comme offrir un exemple d'un individu. L'on ne peut pas offrir un exemple d'un mot parce que, si l'on essaie, l'on se retrouve directement avec ce mot-là. Si cela est vrai, il s'ensuit qu'un mot-type n'est pas un universel, mais bien un individu (eidétique).

\section{Catégorisation multiple}

La deuxième notion que nous introduirons est celle de catégorisation multiple. Pour ce faire, revenons aux exemples (1) et (2). Face à ces exemples, l'on peut tracer deux distinctions emboîtées.

L'on peut opposer les exemples (1) aux exemples (2) : cette distinction est binaire, implique un saut de suppositio et identifie deux dimensions perpendiculaires de catégorisation. Nous les étiquetterons dimension (de catégorisation) eidétique et dimension (de catégorisation) ostensive (\$2.1). 
A l'intérieur de (1) et de (2), l'on peut opposer (1a) à (1b) et (2a) à (2b) : cette distinction est scalaire, est interne à chaque dimension de catégorisation et identifie un continuum de catégorisations spécifiques. A ce propos, nous parlerons de catégorisation multiple eidétique et de catégorisation multiple ostensive $(\S 2.2)$.

La catégorisation multiple ostensive est la notion de catégorisation pertinente pour notre discours.

\subsection{Dimension eidétique vs. Dimension ostensive}

\subsubsection{Dimension eidétique}

Les exemples (1) étaient les suivants :

(1) a. Son roman a été déchiré.

b. Son roman est plein de suspense.

Face à (1), l'on peut affirmer que le mot roman est employé pour sa signification : pareillement, si nous dépensons un billet de cent euros dans un restaurant, nous l'employons pour sa valeur économique et si nous critiquons une thèse de doctorat, nous l'employons pour son contenu. Le mot, le billet de cent euros et la thèse sont envisagés dans une dimension eidétique (ou en suppositio formalis).

La dimension eidétique est illustrée par l'anaphore suivante :

(8) a. J'ai vu un chien. Ce chien était galeux.

Dans (8a), un chien introduit un référent discursif; ce chien, comme Conte (1999a) le souligne, ne reprend pas ce référent directement, mais indirectement : par le biais de la signification du groupe nominal un chien. Puisque une signification est impliquée, nous sommes dans la dimension eidétique.

\subsubsection{Dimension ostensive}

Les exemples (2) étaient les suivants :

(2) a. Roman est un nom commun.

b. Roman est un compliment (pour ce qu'il a écrit).

Face à (2), nous sommes confrontés au même mot roman qu'en (1), mais l'on ne peut plus affirmer qu'il est employé pour sa signification ${ }^{6}$ : de même, si un collectionneur affiche un billet de cent euros dans son album, il ne l'emploie pas pour sa valeur économique, et si un professeur utilise une thèse de doctorat pour caler une table, il ne l'emploie pas pour son contenu. Cette fois, le mot, le billet de cent euros et la thèse sont envisagés dans une dimension ostensive (ou en suppositio materialis).

La dimension ostensive est illustrée par les déixis méta-linguistique et méta-textuelle (cf. Conte 1999c) comme la suivante :

b. Chien. Ce mot est d'origine latine.

Dans (8b), le groupe nominal ce mot se réfère directement au mot chien écrit auparavant, sans passer par la médiation de son sens. Autrement dit, le mot chien n'introduit pas un référent discursif: il est le référent de ce mot. Puisque la signification de chien est contournée, nous sommes dans la dimension ostensive.

\subsubsection{Conclusion}

La différence entre les dimensions eidétique et ostensive peut être appréciée par deux remarques.

Premièrement, comparons $(9 \mathrm{a})$ avec $(9 \mathrm{~b})$ : 
(9) a. J'ai vu un chien. Il était galeux.

b. *Chien. Il est un mot d'origine latine.

En (9a), où la reprise fonctionne, le pronom il peut reprendre un référent discursif introduit par la signification de chien; en (9b), où la reprise échoue, la signification de chien n'introduit aucun référent discursif que le pronom il puisse reprendre ${ }^{7}$.

Deuxièmement, comparons (10a) avec (10b) :

(10) a. *J'ai n'ai pas vu de chien. Ce chien était galeux.

b. Je n'ai pas entendu chien. Ce mot est d'origine latine.

Dans (10a), la négation du prédicat empêche que la signification du groupe nominal un chien puisse être interprétée d'une façon référentielle : l'échec de la reprise montre donc que l'anaphore était sensible à cette signification. Dans (10b), la négation du prédicat n'a aucun effet sur la signification de chien: le fonctionnement de la reprise montre donc que la déixis (méta-linguistique) était indépendante de cette signification.

\subsection{Catégorisation multiple eidétique vs. Catégorisation multiple ostensive}

\subsubsection{Catégorisation multiple eidétique}

Revenons aux exemples (1) en comparant (1a) avec (1b) :

(1) a. Son roman a été déchiré.

b. Son roman est plein de suspense.

En (1a), les prédicats paraissent 'toucher' des aspects différents du concept de roman : déchirer 'touche' le roman en tant qu'objet matériel en papier, alors que être plein de suspense touche le roman en tant que narration. A ce propos, Langacker (1991) parlerait de différentes zones du sujet activées par le prédicat; Nølke (1994) parlerait de différents sèmes du sujet focalisés par le prédicat ; Pustejovsky (1995) parlerait de différent qualia du sujet concernés par le prédicat ; Cruse (1996) parlerait de différentes facettes du sujet illuminées par le prédicat; Kleiber (1999) y verrait un cas de métonymie intégrée. L'intuition derrière ces analyses est que la même entité peut faire l'objet d'une catégorisation multiple. En l'espèce, cette catégorisation a lieu à l'intérieur du contenu du mot roman, dans une dimension eidétique. Il s'agit d'une catégorisation multiple eidétique.

La catégorisation multiple eidétique peut être illustrée à travers des reprises (anaphoriques) catégorisantes qualifiables de internes à la signification:

(11) a. J'ai vu un chien. Cet animal était galeux.

b. J'ai vu un chien. Cette bête était galeuse.

c. J'ai vu un chien. Ce monstre était galeux.

En (11), les reprises soulignées concernent le référent discursif introduit par la signification du mot chien : (11a) offre un hyperonyme du sens de chien, c'est-à-dire l'espèce à laquelle appartient son référent. Ces reprises sont donc internes à la dimension eidétique. Si l'on remplace les 'reprises' (cet animal, etc.) avec ce qui est repris (un chien), l'on obtient des prédications qui restent à l'intérieur de la dimension eidétique : à savoir, Un chien était galeux.

\subsubsection{Catégorisation multiple ostensive}

Comparons maintenant (2a) avec (2b) : 
(2) a. Roman est un nom commun.

b. Roman est un compliment (pour ce qu'il a écrit).

En (2a) et (2b), les prédicats 'touchent' différents aspects du 'sujet' : être un nom touche le mot roman en tant qu'item lexical, alors qu'être un compliment touche le mot roman en tant que résultat d'un choix communicatif. Nous sommes à nouveau confrontés à un phénomène de catégorisation multiple. Cependant, cette catégorisation a lieu à l'extérieur du contenu du mot roman, dans une dimension ostensive. Il s'agit d'une catégorisation multiple ostensive.

La catégorisation multiple ostensive peut être illustrée à travers des reprises (déictiques) catégorisantes qualifiables de externes à la signification:

(12) a. Chien. Ce cri résonnait dans la caserne.

b. Chien. Cet insulte provenait du bureau du colonel.

c. Chien. Ce nom commun vient du latin.

En (12), encore une fois, nous sommes confrontés à des reprises. Cependant, ces reprises ne concernent pas le concept dénoté par chien, mais bien le mot chien dans sa totalité : (12c) ne présente pas l'hyperonyme de ce mot, mais bien l"espèce' à laquelle le mot lui-même appartient. Ces reprises sont donc externes à la dimension eidétique ${ }^{8}$. Si l'on remplace les reprises (ce cri, etc.) avec ce qui est repris (chien), l'on obtient des prédications qui sortent de la dimension eidétique : à savoir, 'Chien' résonnait dans la caserne, 'Chien' provenait du bureau du colonel, 'Chien' vient du latin.

\subsubsection{Conclusion}

Les exemples (1) et (2) sont parallèles car ils présentent le même phénomène de catégorisation multiple qui s'oppose, en bloc, à la polysémie.

La polysémie n'est pas le phénomène par lequel plusieurs prédicats illuminent plusieurs aspects du même objet, mais le phénomène par lequel le même mot saisit plusieurs objets, dont chacun est ouvert, à son tour, à une catégorisation multiple. La polysémie d'un mot (aile d'un oiseau vs. aile d'un édifice) présuppose différents 'objets', alors que la catégorisation multiple d'un objet (roman en tant que narration vs. roman en tant que papier) présuppose un seul et même 'objet'.

Puisque la polysémie présuppose différents 'objets', elle produit un conflit conceptuel :

a. *L'aile était sans plumes et en ruine.

Puisque la catégorisation multiple présuppose un seul 'objet', elle ne produit aucun conflit conceptuel :

b. J'ai lu et déchiré le roman.

c. Roman est un nom commun et un compliment (pour son travail).

Effectivement, les énoncés $(13 b-c)$ ne présentent aucun conflit conceptuel ${ }^{9}$. Dans les deux cas, nous ne sommes donc pas confrontés à un phénomène de polysémie, mais de catégorisation multiple d'un objet. La différence entre (13b) et (13c) consiste seulement dans la dimension où cette catégorisation a lieu : eidétique (13b) vs. ostensive (13c).

La catégorisation multiple ostensive est celle qui est pertinente pour la vocation ostensive du langage. Le contraste avec la catégorisation multiple eidétique, que nous avons conduit tout au long du $\S 2$., nous a permis justement de l'isoler.

\section{Vocation ostensive du langage}

Nous sommes maintenant en mesure d'introduire la notion de vocation ostensive du langage et, par là, notre interprétation de la notion d'autonymie. Avec l'étiquette vocation ostensive du langage, nous 
entendons l'aptitude des contenus linguistiques à s'accrocher aux objets présents dans une situation d'énonciation. Ces objets peuvent être envisagés selon différentes catégorisations ostensives spécifiques. Ces objets, par ailleurs, peuvent être linguistiques ou non linguistiques.

Sous §3.1., nous considérerons le cas où la vocation ostensive s'exerce sur des objets linguistiques, catégorisés ostentivement en tant que : $a$ ) actes communicatifs $(\$ 3.1 .1) ; b)$ actes de composition textuelle $(\S 3.1 .2) ; c)$ choix discursifs $(\S 3.1 .3) ; d)$ œuvres textuelles $(\$ 3.1 .4) ; e$ ) items lexicaux (§3.1.5). Sous $\S 3.2$. , nous considérerons le cas où la vocation ostensive s'exerce sur des objets non-linguistiques.

Avant de rentrer dans les détails, arrêtons-nous un instant sur les listes $(\alpha)$ et $(\beta)$ suivantes :

(a) Il ont lavé la rue

Sérieusement, ie compte sur toi.

Joël est un prénom breton.

Pour résumer. [titre d'un paragraphe d'un article]
(B) Le trottoir est mouillé car ils ont lavé la rue.

\section{Il a a fait son trava ail sérieusement.}

Joël est allé en Bretagne pour le week-end.

J'ai fait $\underline{u}$ un schéma pour résumer.

En $(\beta)$, nous sommes confrontés à des liens internes à la dimension eidétique : les contenus linguistiques des parties soulignées en continu se branchent à ceux des parties soulignées en tirets. En revanche, en $(\alpha)$, nous sommes confrontés à des liens qui sortent de la dimension eidétique : les contenus linguistiques des parties soulignées en continu se branchent non pas aux contenus linguistiques des parties soulignées en tirets, mais bien à ces parties envisagées selon les catégorisations $a$ ) (les deux premiers exemples),e) et $d$ ) susmentionnées. C'est la vocation ostensive du langage. Les exemples $(\alpha)$ sont aussi naturels que $(\beta)$ : en aucun cas une malformation n'est perçue.

\subsection{Objets linguistiques}

\subsubsection{Actes communicatifs}

Considérons les exemples $(14)^{10}$ :

(14) a. Si tu as soif, il y a du champagne dans la cave.

b. Elle n'est pas rentrée, car sa voiture n'est pas garée.

c. Franchement, je suis fatigué.

Dans (14), les contenus linguistiques de si tu as soif, car sa voiture n'est pas garée et franchement ne s'appliquent pas aux contenus linguistiques de il y a du champagne dans la cave, elle n'est pas rentrée et je suis fatigué, mais bien aux messages véhiculés par ces propositions (dimension ostensive). Ces dernières sont catégorisées en tant qu'actes communicatifs : à savoir, offre, hypothèse et plainte.

La vocation ostensive s'exerce ici à un niveau méta-communicatif (cf. Pecorari, sous presse) ${ }^{11}$.

Remarquons qu'en (14a), par exemple, il n'y a aucun besoin de si tu as soif pour déclencher la catégorisation ostensive de il y a du champagne dans la cave : non seulement n'importe quel autre moyen (graphique, phonique, expressif) de mise en évidence aurait suffi, mais (comme Pecorari, sous presse, le souligne) cette proposition aurait pu être introduite déjà dans une telle catégorisation. Les mêmes remarques s'appliquent à tous les autres exemples que nous présenterons.

\subsubsection{Actes de composition textuelle}

Considérons les exemples (15) :

(15) a. Or, depuis l'arrivée au pouvoir des partis islamistes à la faveur des révolutions arabes, les élites économiques anciennes instruisent un procès méthodique en incompétence à l'endroit des nouveaux venus ; ces derniers mettent en avant la légitimité des urnes qui les a portés au 
pouvoir. En somme, les élites nouvelles sont accusées d'incompétence et les anciennes d'illégitimité.

b. On peut établir une analogie quand on perçoit, entre deux réalités différentes, un modèle commun ou une identité de fonctionnement. Par exemple, le corps humain et l'État, quoique correspondant à des réalités différentes, peuvent être rapprochés par analogie [...]

De même qu'en (14), dans (15), les contenus linguistiques de en somme et par exemple ne s'appliquent pas aux contenus linguistiques des propositions qui les suivent. Différemment de (14), en (15), en somme et par exemple ne s'appliquent pas à ces propositions catégorisées en tant qu'actes de communication, mais bien en tant qu'actions de composition textuelle : à savoir, une synthèse et une exemplification.

La vocation ostensive s'exerce ici à un niveau de l'organisation méta-textuelle.

\subsubsection{Choix discursifs}

Considérons les exemples (16) :

(16) a. Le pape François est très cool, si ce mot convient à un pape.

b. Tout a commencé dans la maison de ses parents, plus exactement dans leur chambre à coucher.

Pour (16a) Rey-Debove (1978/1997) et Authier-Revuz (1995) parlent de connotation autonymique, De Brabanter (2003) parle de hybrides et Recanati (2001, 2010) parle de citation ouverte. En (16), cool et chambre à coucher sont présents avec une double catégorisation ou une catégorisation croisée ${ }^{12}$ : ils sont employés pour leur signification par être cool et commencer (dimension eidétique); et ils sont catégorisés en tant que résultats d'un choix discursif du locuteur (dimension ostensive) par si ce mot convient et plus exactement.

D'abord, comparons (16) avec (14) et (15). Nous sommes confrontés au même type de phénomène. La différence spécifique de (16) est que les mots jouissent d'un double statut (eidétique et ostensif). Par ailleurs, en (14), la vocation ostensive s'exerce au niveau méta-communicatif, alors qu'en (16), elle s'exerce au niveau méta-textuel.

Ensuite, comparons les exemples (16) entre eux. Ils diffèrent par le critère de l'évaluation du choix discursif : en (16a), il est question d'adéquation interpersonnelle ; en (16b), il est question d'adéquation idéative. En ce qui concerne (16b), l'on remarquera que la catégorisation ostensive de chambre à coucher impose une rétro-catégorisation ostensive également sur maison : par conséquent, bien que le contenu de chambre à coucher soit un rapport syntagmatique avec le contenu de maison, les mots maison et chambre à coucher sont présentés comme étant en opposition (donc, en rapport paradigmatique).

\subsubsection{OEuvres textuelles}

Considérons les exemples (17) :

(17) a. Pour une logique du sens.
[titre d'un livre]
[dédicace d'une thèse de doctorat]

b. A mes parents

L'exemple (17a) est le titre d'un livre de R. Martin, alors que l'exemple (17b) est la dédicace d'une thèse de doctorat. Évidemment, les contenus linguistiques de (17) ne s'appliquent pas aux contenus textuels, mais au texte lui-même. Cependant, le texte n'est catégorisé ni en tant qu'acte communicatif, ni en tant qu'acte de composition textuelle, ni en tant que résultat d'un choix discursif, mais bien en tant qu'œuvre.

Comparons (17a) avec (14a) et (18) :

(18) Aujourd'hui, je suis rentré plus tôt pour passer au pôle emploi.

En (18), pour passer au pôle emploi exprime un but : ce but s'accroche au contenu propositionnel de la principale et, avec les termes de Prandi (2004:279), nous sommes confrontés à une subordination 
intégrée. De même, en (14a) et en (17a), si tu as soif et pour une logique du sens expriment un but ; cependant, contrairement à (18), ces buts ne s'accrochent pas aux contenus linguistiques des propositions principales. Avec les termes de Prandi (2004:279), nous sommes confrontés à des subordinations non intégrées. La différence entre (14a) et (17a) est que l'objet linguistique auquel si tu as soif s'accroche est un message, alors que l'objet linguistique auquel pour une logique $d u$ sens s'accroche est un livre.

La vocation ostensive s'exerce ici au niveau des œuvres.

\subsubsection{Items lexicaux}

Considérons maintenant l'exemple (19) :

(19) Ange a quatre lettres.

Le mot Ange est présent et catégorisé (ostensivement) en tant que type ou item lexical. La vocation ostensive s'exerce ici au niveau métalinguistique. Par rapport aux cas précédents, (19) identifie un point zéro.

Dans les exemples (14)-(17), la signification des mots en gris fonctionne comme motivation des actes communicatifs, de composition textuelle, de choix discursifs, etc. accomplis grâce à eux. En (14a), l'offre du champagne est motivée par la signification de la principale qui explicite l'une de ses conditions de réussite (i.e. le fait qu'il y a du champagne). En (15a), l'action de synthèse (introduite par en somme) est motivée par la relation (de spécification) entre la signification de la portion textuelle en gris et la signification de portion textuelle précédente. En (16a), le choix discursif du mot cool s'avère motivé par l'aspect interpersonnel de la signification de ce mot. Des remarques semblables sont valables pour les autres exemples. Même dans le cas limite du livre, le type textuel (essai de linguistique, thèse de doctorat) s'avère motivé par le contenu du texte.

En (19), en revanche, la signification du mot Ange est complètement mise entre parenthèses. La catégorisation ostensive ne nous présente ni un acte linguistique (communicatif, de composition textuelle, etc.), ni un type de texte, mais elle 'recule' au point zéro du pur objet lexical partagé par les francophones.

\subsection{Objets non linguistiques}

Considérons encore les exemples suivants :

(20) a. Parce que tu es belle.

b. Madame, si vous voulez vous asseoir

c. Fabriqué en Chine. [en offrant une rose]

[en libérant une place]

[imprimé sur une tasse]

Imaginons que (20a) soit prononcé en offrant une rose, que (20b) soit énoncé en laissant libre une place sur le métro pour une femme enceinte et que (20c) soit imprimé sur la anse d'une tasse. Si nous comparons ces exemples avec les précédents, nous remarquons un bouleversement : nous ne sommes plus confrontés à la présence du langage, mais à la présence du monde en général. En (20a-b), il y a des actions (faire un cadeau et offrir sa place), mais elles ne sont pas linguistiques; et en (20c) il y a un objet matériel. Ainsi, les contenus linguistiques en (20) s'accrochent à des objets extralinguistiques : la vocation ostensive s'exerce donc sur le monde.

Dans un énoncé tel que Je t'offre cette rose parce que tu es belle, la subordonnée s'attache à la proposition je t'offre cette rose; en (20a), en revanche, la même subordonnée s'attache directement à la fleur (ou au geste de l'offrir). 


\subsection{Conclusion}

Résumons notre démarche en nous concentrant sur la notion d'autonymie. Sous $§ 1$, nous avons soutenu que les mots sont les seuls objets nécessairement présents dans le champ d'indication identifié par une situation énonciative concrète. Sous $\S 2$, nous avons soutenu que les mots (nécessairement exhibés) peuvent faire l'objet d'une catégorisation multiple ostensive. Sous $\S 3$, nous avons distingué différents types de catégorisations ostensives, déclenchées sur des mots par les contenus d'autres mots qui s'y accrochent (avec le caveat du \$3.1.1). Le phénomène de l'autonymie s'est avéré comme l'un de ces types. Nous avons appelé vocation ostensive du langage l'aptitude des structures linguistiques à s'accrocher à tout objet (linguistique ou extralinguistique) présent dans une situation d'énonciation et envisagé selon une catégorisation ostensive spécifique.

Focalisons-nous sur les niveaux de catégorisation ostensive considérés sous $\S \S 3.1$. et 3.2.. Ces niveaux peuvent être conçus comme des cercles qui s'éloignent d'un centre idéel, identifié par le contenu linguistique : $i$ ) niveau méta-communicatif: offres, conseils, affirmations ; ii) niveau méta-textuel : reformulations, exemplifications, commentaires sur l'adéquation idéative ou interpersonnelle des mots ; iii) niveau des œuvres textuelles : titres, dédicaces ; iv) niveau méta-linguistique : items lexicaux.

Le niveau $i v$ ) trace la limite entre l'exercice de la vocation ostensive sur des objets linguistiques et son exercice sur des objets non linguistiques. Ainsi, la vocation ostensive du langage peut être envisagée comme un vecteur qui part du centre idéel du contenu propositionnel, traverse tous ces cercles, et dépasse les bornes du métalinguistique pour toucher directement au monde extralinguistique ${ }^{13}$

En gardant le langage comme point de repère, nous pouvons distinguer deux directions pour la vocation ostensive : endocentrique, si les objets sur lesquels elle s'exerce sont linguistiques ; et exocentrique, dans le cas contraire. La direction endocentrique est à la base des phénomènes exemplifiés sous $\$ 3.1$, alors que la direction exocentrique montre que le monde n'offre pas seulement un support au langage (comme un mur pour des affiches), ni seulement un dépôt de référents, mais qu'il rentre directement dans le langage.

\section{F. A. Q. concernant l'autonymie}

Parmi les frequently asked questions concernant l'autonymie, l'on peut sans doute compter les suivantes : a) Un énoncé contenant un autonyme est-il mal formé ? $(\S 4.1)$; b) Quel est le statut linguistique (nom, nom propre, etc.) d'un autonyme ? $(\S 4.2) ; c)$ Les guillemets sont-ils des démonstratifs ? (\$4.3); d) L'autonymie est-elle un phénomène de représentation (citation, mimesis, etc.) ? (\$4.4.) ; e) La dichotomie usage vs. mention correspond-t-elle au phénomène de l'autonymie ? (§4.5).

\subsection{Un énoncé contenant un antonyme est-il mal formé ?}

Un énoncé peut être mal formé sémantiquement ou syntaxiquement : dans le premier cas, nous sommes confrontés à une incohérence (cf. Conte 1999b); dans le second cas, nous sommes confrontés à une inconsistance (Conte 1999b). Ainsi, notre question se divise en deux : al) un énoncé contenant un autonyme est-il incohérent ? $(\$ 4.1 .1) ; a 2)$ un énoncé contenant un autonyme est-il inconsistant ? (\$4.1.2).

\subsubsection{Un énoncé contenant un autonyme est-il incohérent ?}

Dans un poème, il peut y avoir la métaphore suivante :

(21) a. L'arbre a chuchoté un rouge gorge.

Face à (21a), nous imaginons un rouge-gorge qui sort du feuillage d'un arbre comme un mot de la bouche d'une personne. Ici, le mot rouge-gorge est utilisé pour sa signification et occupe la position d'objet direct : nous sommes dans la dimension eidétique. Considérons maintenant (21b) :

b. L'enfant a chuchoté un rouge-gorge. 
Cette fois, la signification du mot rouge-gorge est mise entre parenthèse : ainsi, rouge-gorge peut remplir une fonction semblable à un objet direct, mais il n'est pas, stricto sensu, un objet direct. L'énoncé (21b) est comparable à une tasse avec l'écriture fabriqué en Chine : nous sommes dans la dimension ostensive. Le point crucial est que l'opposition entre cohérence et incohérence conceptuelle (non métaphore $v s$. métaphore) est pertinente exclusivement à l'intérieur de la dimension eidétique ${ }^{14}$. La question que pose le titre est donc hors de propos.

Une conséquence est qu'un autonyme n'est ni un cas de polysémie, ni un cas d'homonymie. Il ne s'agit pas d'un cas de polysémie : la polysémie a lieu dans la dimension eidétique et, en (21b), nous sommes dans la dimension ostensive ${ }^{15}$. Il ne s'agit non plus d'un cas d'homonymie : l'homonymie présuppose deux mots jumeaux (avec un signifiant identique et un signifié différent), mais en (21) nous sommes confrontés au même mot rouge-gorge, différemment catégorisé.

\subsubsection{Un énoncé contenant un autonyme est-il inconsistant ?}

Considérons l'exemple (22a) :

(22) a. Mais est une conjonction.

Selon De Brabanter (2005:25), un exemple comme (22a) nous présenterait une impasse : soit l'on admet qu'une phrase puisse avoir la structure Conjonction + Groupe Verbal (à la place de Groupe Nominal + Groupe Verbal), soit l'on admet qu'une conjonction puisse se 'transformer' en groupe nominal. Mais, évidemment, aucun de ces deux chemins ne peut être emprunté.

Ajoutons à (22a), les exemples (22b) et (22c) :

(22) b. Le chat est passé par la cuisine, car l'assiette de saucisses est vide.

c. Fabriqué en Chine [imprimé sur une tasse]

Dans (22), nous sommes confrontés à la même espèce de phénomènes basés sur la vocation ostensive du langage (cf. §3). Cela n'implique nullement qu'il n'y a pas de différences.

En (22b), la subordonnée ne s'accroche pas au contenu de la principale, mais bien à l'acte linguistique accompli par elle. En (22a), le prédicat ne s'accroche ni au contenu de mais, ni à un acte linguistique, mais bien à mais envisagé comme item lexical, dont la signification est mise entre parenthèse. Par conséquent, (22a) est plus extrême que (22b) : plus éloigné du centre des cercles envisagés sous §3.3. Quant à (22c), cet exemple est encore plus extrême que (22a). En (22a), le mot mais est employé dans une dimension ostensive, mais il reste un mot: les contenus du prédicat ne s'accrochent pas au contenu de mais, mais ils s'accrochent quand-même à un objet linguistique. En (22c), en revanche, les contenus linguistiques s'accrochent à un objet non linguistique. Cela explique pourquoi un prédicat actualisé comme est une conjonction s'attache avec naturel au mot mais, alors qu'un prédicat actualisé comme $a$ été fabriqué en Chine imprimé sur une tasse nous paraîtra bizarre et l'on préférera la version non actualisée fabriqué en Chine.

\subsection{Quel est le statut linguistique d'un autonyme?}

Quand l'on s'interroge sur la partie du discours d'un autonyme, la réponse classique est qu'il s'agit d'un nom propre sui generis ${ }^{16}$. A ce propos, Petit (2009:223-225) offre une discussion détaillée. S'interroger sur la partie du discours d'un autonyme présuppose que les signes autonymique et non autonymique sont des entités différentes. Or, cette présupposition est fausse.

Imaginons à nouveau une tasse avec l'écriture fabriqué en Chine. Par rapport à cet énoncé, la tasse se comporte comme un nom propre car elle occupe une position thématique semblable à celle de sujet. Mais il serait absurde de s'interroger sur sa partie du discours, parce qu'il s'agit... d'une tasse !

Revenons à (22a) : 
(22) a. Mais est une conjonction.

Dans cet exemple, le mot mais fonctionne comme la tasse : il occupe une position thématique semblable à un sujet. Cependant, contrairement à la tasse, mais est un mot. En tant que mot, sa partie du discours est : conjonction. Voici la réponse à la question de ce paragraphe.

\subsection{Les guillemets sont-ils des démonstratifs ?}

La thèse, très influente, du philosophe Davidson (1979) est que les guillemets sont effectivement des démonstratifs.

La différence entre un vrai démonstratif (par exemple le pronom ce) et les guillemets est que le premier a une signification, alors que les seconds non. Comparons l'espace entre les guillemets " " avec la signification du pronom $c e$, qui peut être paraphrasée, grosso modo, comme : «l'objet auquel le locuteur se réfère en disant $c e »$. Ils ont une caractéristique commune et une différence. La caractéristique commune est que la circularité de la signification du pronom remplit la même fonction de l'espace vide entre les guillemets : elle permet au pronom d'être déictique (en soutenant donc l'intuition de Davidson). La différence est que cette circularité est une signification codée par le lexique, alors que l'espace entre les guillemets est une portion réelle du champ déictique.

Si cela est vrai, les guillemets sont nécessairement des outils méta-textuels, alors que les démonstratifs sont des items lexicaux. Autrement dit, les guillemets sont semblables à la tonalité de la voix ou à un clin d'œil : une sorte de face games textuels pour mettre en évidence quelque chose sur le champ déictique (cf. §1.2). Évidemment, la raison pour laquelle l'on met en évidence quelque chose (par exemple, problématiser un certain choix discursif) ne peut pas être codée a priori, mais elle peut seulement être inférée à chaque acte de parole, sur la base de la cohérence textuelle (au sens de Conte 1999b).

\subsection{L'autonymie est-elle un phénomène de représentation ?}

Cette question peut être clarifiée en la décomposant en deux : $d 1$ ) L'autonymie relève-t-elle de la citation ? $(\S 4.4 .1) ; d 2)$ L'autonymie est-elle un phénomène de mimesis ou d'iconicité ? (\$4.4.2).

\subsubsection{L'autonymie relève-t-elle de la citation ?}

Considérons les exemples suivants :

(23) a. Ange est un nom commun de quatre lettres.

b. Il portait un chapeau, si ce mot convient à la soucoupe volante qu'il avait sur la tête.

Selon Recanati (2001), en (23a) nous sommes confrontés à un cas de citation fermée (bien que limite), alors qu'en (23b) nous sommes confrontés à un cas de citation ouverte. Cependant, s'il s'agit de citations, qui cite-t-on? Selon le sens commun, il ne s'agit pas de citations car ces mots ne sont attribués à aucun locuteur.

En $(23 \mathrm{c})$, en revanche, il y a une citation :

(23) c. Ce garçon est une crème, comme le dit ma mère.

En (23c), le mot crème a une double catégorisation, en dimension eidétique et en dimension ostensive. En (23a) et (23c) les mots sont à la fois présentés et objets de discours. La différence concerne la façon dont on parle de ces mots : en (23a), on les catégorise en tant qu'items lexicaux, alors qu'en (23c) on les catégorise en tant que mots prononcés par quelqu'un. 


\subsubsection{L'autonymie est-elle un phénomène de mimesis ou d'iconicité ?}

Si l'autonymie (selon le sens commun) ne relève pas de la citation, relève-t-elle d'une forme de mimesis ou iconicité ? A notre avis, encore une fois, la réponse est négative. Toute représentation ou mimesis présuppose une différence de type entre icône et original, mais en (23a) nous sommes confrontés à l'original lui-même.

Sur le célèbre tableau de Magritte, il est écrit : ceci n'est pas une pipe. Si nous entendons cette phrase comme: ceci n'est pas une vraie pipe, elle exprime l'évidence permettant au dessin d'être la représentation d'une pipe. Autrement dit, la condition à laquelle un 'objet' peut être l'icône d'un original est qu'il ne soit pas cet original. Le dessin d'une pipe, n'est pas une pipe, mais un dessin ; la reproduction en bois d'un fusil, n'est pas un fusil, mais un objet en bois. La même remarque est valable pour les actions. Supposons que l'un de nos amis ait une drôle de façon de marcher: nous pouvons imiter ou caricaturer sa façon de marcher car notre action ne sera pas une occurrence de sa façon de marcher. Supposons que nous ayons une drôle de façon de marcher : nous pouvons imiter ou caricaturer notre propre façon de marcher dans la mesure où l'action que nous allons accomplir ne comptera pas comme une vraie occurrence de notre (drôle de) façon de marcher. Ces remarques excluent, nous semble-t-il, que l'autonymie puisse être considérée comme un cas de mimesis.

$\mathrm{Si}$, face à enge, nous étions confrontés à une icône qui ne ressemble pas à son original (ange), l'on pourrait effectivement soutenir que, face à ange, nous sommes confrontés à une icône parfaitement ressemblante à l'original. Mais il est clair que enge comme ange sont le même original ange: dans un cas, cet original est mal écrit, alors que dans l'autre il est bien écrit. Une icône, en revanche, n'est pas évaluée par rapport à la dimension erroné vs. correct. Comme le montre l'exemple du sage Balnibarni, en (23a), ange n'est pas une imitation de l'item lexical ange $:$ il est cet item.

En somme, l'on ne peut pas imiter un mot car, ce faisant, nous disons ce mot-là. C'est pour cette même raison que nous ne pouvons pas exemplifier un mot (cf. §1.3.) et que, pour les mots, l'astuce de Magritte ne fonctionne pas :

*Chat. Ceci n'est pas un mot, mais seulement sa représentation.

\subsection{La dichotomie usage vs. mention correspond-t-elle au phénomène de l'autonymie?}

Le couple usage vs. mention souligne que le phénomène de l'autonymie n'est pas un cas d'emploi (usage) d'un mot. Ici, nous sommes parfaitement d'accord. L'on peut parler d'une façon rigoureuse d'emploi d'un mot exclusivement au niveau de la catégorisation eidétique : là où il y a le phénomène de la polysémie. Mais le couple usage vs. mention place l'autonymie sous la rubrique mention. Or, le TLFI définit mention comme : "Action de faire remarquer ou connaître (par la parole ou par un écrit), de citer ». Ici, la question est délicate.

Tout d'abord, revenons à un exemple tel que (25a) :

\section{a. Chien. Ce nom est d'origine latine.}

Si le mot dit autonymique est conçu comme le sujet de l'action de mentionner, alors la notion de mention ne s'applique pas. En effet, dans (25a), chien ne mentionne rien, mais il est simplement lui-même. Si, en revanche, le mot dit autonymique est conçu comme un objet présent dans le champ d'indication, mentionné par d'autres mots (en l'espèce, ce nom), alors la notion de mention a un (tout petit) espace d'application.

Ensuite, revenons à un exemple tel que (25b) :

$$
\text { b. Ange a quatre lettres. }
$$

Si le mot dit autonymique est conçu comme le sujet de l'action de mentionner, notre conclusion répète la précédente. Cependant, cette fois, l'on ne peut pas concevoir le mot autonymique comme objet 
mentionné car, en (25b), il n'y a aucun mot qui accomplit l'action de mentionner (cf.§1.2). En (25b), il y a seulement le prédicat avoir quatre lettres qui s'attache au mot Ange, ostensivement catégorisé comme item lexical et mis en évidence par l'italique. Cette fois, la notion de mention n'a aucun espace d'application.

\section{Références bibliographiques}

Authier-Revuz, J. (1987). Modalité autonymique et pseudo anaphore déictique. Cahiers de lexicologie, 51, 19-37.

Authier-Revuz, J. (1995). Ces mots qui ne vont pas de soi. Boucles réflexives et non-coïncidence du dire (Vol. 1\&2) Paris : Larousse.

Authier-Revuz, J. (2003). Le Fait autonymique : Langage, langue, discours. Quelques repères. En : Authier-Revuz J., Doury M, Reboul-Touré, S. (éd.). Parler des mots. Le fait autonymique en discours. Paris : Presses Sorbonne Nouvelle, 67-96.

Christensen, N. E. (1967). The alleged distinction between use and mention. Philosophical Review, 76, 358-67.

Conte, M-E. (1999a). Deissi testuale e anafora. In : Conte, M-E.. Condizioni di coerenza. Ricerche di linguistica testuale. Alessandria : Edizioni dell'Orso, 11-28.

Conte, M-E. (1999b). Coerenza testuale. In : Conte, M-E.. Condizioni di coerenza. Ricerche di linguistica testuale. Alessandria : Edizioni dell’Orso, pp. 29-46.

Conte, M-E. (1999c). Metatestualità. In : Conte, M-E.. Condizioni di coerenza. Ricerche di linguistica testuale. Alessandria : Edizioni dell'Orso, 47-50.

Cruse D. A. (1996). La signification des noms propres de pays en anglais. En : Rémi-Giraus, S. \& Rétat, P. (ed.). Les mots de la nation. Lyon : Presses Universitaires de Lyon.

Davidson, D. (1979). Quotation. Theory and Decision, 11, 27-40.

De Brabanter, Ph. (ed.) (2003). Hybrid Quotations, Belgian Journal of Linguistics, 17.

De Brabanter, $\mathrm{Ph}$. (2005). Philosophes du langage et autonymie : une déjà longue histoire. Histoire Epistémologie Langage, 27/1, 11-43.

Fasciolo, M. (2008). Due interrogativi sull'expressione delle relazioni concettuali. In : Ferrari, A. (ed.). Sintassi storica e sincronica dell'Italiano. Subordinazione, coordinazione, giustapposizione. Vol. 2. Firenze : Franco Cesati Editore.

Gross, G. (2012). Manuel d'analyse linguistique. Approche sémantico-syntaxique du lexique. Villeneuve d'Ascq: Presses Universitaires du Septentrion.

Gross, G. \& Prandi, M. (2004). La Finalité. Fondements conceptuels et genèse linguistique, Bruxelles : de boeck.duculot.

Jakobson R. (1963). Essais de linguistique générale. Paris : Minuit.

Langacker, R. W. (1991). Concept, Image and Symbol. The Cognitive Basis of Grammar, Berlin, Mouton de Gruyter.

Lyons, J. (1977). Semantics 1. Cambridge : Cambridge University Press.

Kleiber G. (1994). Anaphores et pronoms. Louvain-la-Neuve : Duculot.

Kleiber, G. (1999). Problèmes de sémantique. La polysémie en questions. Villeneuve d'Ascq : Presses Universitaires du Septentrion.

Nølke, H. (1994). Linguistique modulaire. De la forme au sens. Paris : Peeters.

Pecorari, F. (sous presse). Metalinguistico, metatestuale e metacomunicativo. Mezzi di coesione testuale tra anafora e deissi. En : Ferrari A., Korzen I., De Cesare A-M. (ed). Tra romanistica e germanistica: lingua, testo, cognizione e cultura / Between Romance and Germanic: language, text, cognition and culture. Berne :Peter Lang. 
Petit, G. (2009). La dénomination : approches lexicologique et terminologique. Louvain-Paris : Peeters.

Prandi, M. (2004). The building blocks of meaning, Amsterdam/Philadelphia : John Benjamins.

Prandi, M. (2006). Le regole e le scelte. Torino : UTET.

Quine, W. V. O. (1960), Word and Object, Londres, Wiley, Studies in Communication.

Recanati, F. (1979). La transparence et l'énonciation. Pour introduire à la pragmatique, Paris : Seuil.

Recanati, F. (2001). Open quotation, Mind, 110, 637-87. Réimprimé en : Recanati, F. (2010). Truth-conditional pragmatics. Cambridge : Cambridge Univeristy Press, 219-267.

Rey-Debove, J. (1997). Le Métalangage. Étude linguistique du discours sur le langage, 2è édition augmentée. Paris : Armand Colin.

Riegel, M. (1990). La définition, acte du langage ordinaire. De la forme aux interprétations. En : Chaurand J. Mazière F. (éd.). La définition. Paris : Larousse.

Saka, P. (1998). Quotation and the use-mention distinction. Mind, 197, 425, 113-135.

Searle, J. R. (1969), Speech Acts : An Essay in the Philosophy of Language, Cambridge/New York, Cambridge University Press. Tr. fr. : Searle, J. R. $(1972,1996)$. Les actes de langage. Essai de philosophie du langage. Paris : Hermann.

Sørensen, H. S. (1961), An analysis of linguistic signs occurring in suppositio materialis or the meaning of quotation marks and their phonetic equivalents, Lingua, 10-2, 174-89.

Tarski, A. (1944). The semantic conception of truth and the foundations of semantics. Journal of Philosophy and Phenomenological Research, 4, 341-75. Traduit en français par G.-G. Granger en : Tarski, A. (1974). Logique sémantique et mathématique II. Paris : Armand-Colin, 267-305.

Washington, C. (1992), Identity theory of quotation, Journal of Philosophy, 89, 582-605.

Pour la séparation, en (0), il y a donc deux mots-types. Plus précisément, il y a un token du mot-type autonyme en (0a) et un token du mot-type non autonyme en $(0 \mathrm{~b})$. Pour une présentation synthétique de la distinction type/token, cf. Lyons (1977:13).

${ }^{2}$ Pour l'unification, en (0a) et en (0b), il y a deux tokens du même mot-type chat.

3 Certes, l'étiquette théorie de la dénomination a été forgée pour les positions de A. Tarski et W. O. Quine : par conséquent, son application à J. Rey-Debove et à J. Authier-Revuz peut paraitre philologiquement incorrecte. Cependant, reste le fait que les positions de tous ces auteurs partagent l'opposition à la conception de J. Searle. Deux exemples d'auteurs mélangeant des traits du pôle de l'unification et du pôle de la séparation sont, à notre avis, P. Saka (1998) et F. Recanati $(2001,2010)$.

4 D'ailleurs, il n'y a aucun sens à forger une notion générale comme 'Baleine' ou La 'baleine' qui rassemblerait toutes les occurrences du mot baleine, alors qu'il est parfaitement sensé d'imaginer une notion comme Baleine ou La baleine pour rassembler toutes les différentes baleines particulières.

$5 \quad$ Peut-être, l'on peut améliorer (7b) grâce à (7c) : ?Pourriez-vous me montrer un exemple du mot 'baleine', s'il vous plaît? Cependant, la question $(7 \mathrm{c})$ est heureuse seulement en l'interprétant comme la requête non pas d'un exemple d'un mot (ce qui ne signifie strictement rien), mais bien comme la requête d'un exemple de la façon d'employer un mot.

6 Certes, la signification du mot roman est la motivation à la base de l'action de qualifier une certaine œuvre de roman. Cependant, en (2b), le prédicat être un compliment s'applique non pas à la signification du mot roman, mais bien à l'action de qualifier quelque chose de roman (motivée par cette signification).

$7 \quad$ Pour une analyse détaillée du fonctionnement du pronom $i l$, nous renvoyons à Kleiber (1994).

$8 \quad$ Pour une discussion du rapport entre autonymie, anaphore et déixis, l'on verra aussi Authier-Revuz (1985). 
S'il y a un conflit, il s'agit d'un conflit informationnel et non conceptuel : soit à cause de l'ordre de présentation des informations ( ??J'ai brûlé et lu le livre), soit à cause de la cohérence thématique des informations (?Cette thèse de doctorat est mal argumentée et toute tachée).

${ }^{10}$ Nous mettrons en évidence, en gris, les objets linguistiques présents (dans le type de catégorisation ostensive à chaque fois pertinent) et nous soulignerons les contenus linguistiques qui s'y attachent.

11 Un autre exemple (au niveau 'macro communicatif') est offert par des formules telles que cordialement, amicalement, etc. à la fin d'une lettre. Ces adverbes s'attachent à l'acte de s'adresser au destinataire, accompli avec le texte.

12 Riegel (1990:105) remarque ce point avec clarté.

${ }^{13}$ Les mots qui ont fait l'objet des catégorisations ostensives sous $\$ 3.1$. peuvent être définis négativement comme n'étant pas en suppositio formalis. Cependant, peuvent-ils être définis positivement avec l'étiquette unique de suppositio materialis? Peut-être la qualification de matériel est-elle appropriée pour le cas d'un mot envisagé comme cri ou tache d'encre, mais elle ne nous paraît pas appropriée pour les catégorisations méta-communicatives ou méta-textuelles.

${ }^{14}$ Remarquons que pour cette interprétation le changement de sujet n'est pas nécessaire : L'arbre a chuchoté "un rouge-gorge".

${ }^{15}$ Nous ne pouvons donc pas être en accord avec Saka (1998:128-129).

16 Considérer un autonyme comme une sorte de nom propre signifie reconnaitre implicitement qu'un mot-type est un individu $(\S 1.3)$. 